\title{
The Exact Solutions of the Conformable Time-Fractional Modified Nonlinear Schrödinger Equation by the Trial Equation Method and Modified Trial Equation Method
}

\author{
Safoura Rezaei Aderyani $\mathbb{D D}^{1}{ }^{1}$ Reza Saadati $\left(\mathbb{D},{ }^{1} \mathrm{Javad}\right.$ Vahidi $\left(\mathbb{D},{ }^{1}\right.$ \\ and Tofigh Allahviranloo $\mathbb{1}^{2}$ \\ ${ }^{1}$ School of Mathematics, Iran University of Science and Technology, Narmak, Tehran, Iran \\ ${ }^{2}$ Istinye University, Faculty of Engineering and Natural Sciences, Istanbul, Turkey \\ Correspondence should be addressed to Reza Saadati; rsaadati@eml.cc
}

Received 19 November 2021; Revised 3 January 2022; Accepted 7 January 2022; Published 1 February 2022

Academic Editor: Ruben Specogna

Copyright (c) 2022 Safoura Rezaei Aderyani et al. This is an open access article distributed under the Creative Commons Attribution License, which permits unrestricted use, distribution, and reproduction in any medium, provided the original work is properly cited.

Using the trial equation method (TEM) and modified trial equation method (MTEM), firstly, we find the analytical solutions of the conformable time-fractional modified nonlinear Schrödinger equation (CTFMNLSE), and finally, we present numerical results in tables and charts.

\section{Introduction}

In the past ten years, NLSEs have received a great deal of interest from scholars and researchers. Actually, simplified versions of Zakharov's system give rise to certain NLSHs. For more details, see [1]. NLSEs have applications in different subjects, e.g., quantum mechanics, biology, semiconductor industry, optical communication, energy quantization, quantum chemistry, wave propagation, protein folding and bending, condensed matter physics, solid-state physics, nanotechnology and industry, laser propagation, and nonlinear optics.

Lately, the investigation of the CTFNLSE in the form

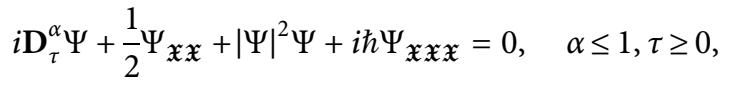

including numerics, analysis, and applications becomes a significant issue in applied mathematics, and diverse computational methods have been designed to discuss the exact solutions of it. Every method has its own proportion formulas and merits for the application to the administering equation for exploring the exact solutions.
In [2-4], the exact solution of CTFNLSE (1) is obtained through different methods such as the first integral method, functional variable method, sine-Gordon method, and direct algebraic method. In [5], Younas et al. presented the CTFMNLSE and studied the exact solutions of it by the generalized exponential rational function method.

Motivated by Younas et al. [5], we consider the following CTFMNLSE:

$$
\begin{aligned}
i \mathbf{D}_{\tau}^{\alpha} \Psi+\sigma_{1} \Psi_{\mathfrak{X X}}+\sigma_{2}|\Psi|^{2} \Psi= & i \delta_{1} \Psi_{\mathfrak{X X X}}+i \delta_{2} \Psi^{2} \Psi_{\mathfrak{X}}^{*} \\
& -i \delta_{\mathfrak{3}}|\Psi|^{2} \Psi_{\mathfrak{X}}+\delta_{4} \Psi, \quad 0<\alpha \leq 1,
\end{aligned}
$$

where $\quad \sigma_{1}=\left(\left(\mathfrak{X}_{0}\right) / 8 \mathscr{K}_{0}^{2}(-3 \cos (\Theta)+2)\right), \sigma_{2}=\left(\left(-\mathfrak{X}_{0}\right.\right.$ $\left.\left.\mathscr{K}_{0}^{2}\right) /(2)\right), \delta_{1}=\left(\left(\mathfrak{X}_{0} \cos (\Theta)\right) /\left(16 \mathscr{K}_{0}^{3}\left(-5 \cos ^{2}(\Theta)-6\right)\right)\right), \delta_{2}$ $=\left(\left(\mathfrak{X}_{0} \mathscr{K}_{0} \cos (\Theta)\right) /(4)\right), \delta_{3}=\left(3 \mathfrak{X}_{0} \mathscr{K}_{0}\right) /(2), \delta_{4}=\mathscr{K}_{0}|\Psi|_{\mathfrak{X}}^{2} \mid$ $\mathfrak{X}=0, \mathfrak{X}_{0}$ and $\mathscr{K}_{0}$ are the frequency and the wave number of the carrier wave, respectively, and the operator $\mathbf{D}^{\alpha}$ of order $\alpha$, where $\alpha \in(0,1]$, represents the conformable fractional derivative.

Here, using the TEM and MTEM, we investigate the exact solutions for the above CTFMNLSE. For more details, you can see [6-11]. 


\section{Preliminaries}

Here, it would be helpful to present some properties and definitions of the conformal derivative and other preliminaries.

Definition 1 (see [12]). Suppose $\Omega:(0, \infty) \longrightarrow \mathbb{R}$ is a function. Then, the conformal fractional derivative of $\Omega$ of order $\alpha$ is presented as

$$
\mathbf{T}_{\alpha}(\tau)=\lim _{\epsilon \longrightarrow 0} \frac{\Omega\left(\tau+\epsilon \tau^{1-\alpha}\right)-\Omega(\tau)}{\epsilon},
$$

for all $0<\alpha \leq 1,0<\tau$.

Definition 2 (fractional integral; see [12]). Suppose $\iota \geq 0$ and $\tau \geq \imath$. Also, suppose $\Omega$ is a function defined on $(\iota, \tau]$ and $0<\alpha<1$. Then, the $\alpha$-fractional integral of $\Omega$ is defined by

$$
\mathbf{I}_{l}^{\alpha} \Omega(\tau)=\int_{\iota}^{\tau} \frac{\Omega(\varsigma)}{\varsigma^{1-\alpha}} \mathrm{d} \varsigma
$$

if the Riemann improper integral exists.

Theorem 1 (see [12]). Suppose $0<\alpha \leq 1$ and $\Omega$ and $\sigma$ are $\alpha$-differentiable at a point $\tau$; then,

$$
\begin{aligned}
& \text { (i) } \mathbf{T}_{\alpha}\left(\varpi_{1} \Omega+\varpi_{2} \delta\right)=\varpi_{1} \mathbf{T}_{\alpha}(\Omega)+\varpi_{2} \mathbf{T}_{\alpha}(\delta), \forall \varpi_{1} \text {, } \\
& \omega_{2} \in \mathbb{R}
\end{aligned}
$$

(ii) $\mathbf{T}_{\alpha}\left(t^{\Phi}\right)=\varpi \tau^{\Phi-\alpha}, \forall \bowtie \in \mathbb{R}$

(iii) ${ }_{\tau} \mathbf{T}_{\alpha}(\Omega \widetilde{\delta})=\Omega \mathbf{T}_{\alpha}(\delta)+\widetilde{\delta} \mathbf{T}_{\alpha}(\Omega)$

(iv) $\mathbf{T}_{\alpha}(\Omega / \widetilde{\delta})=\left(\left(\widetilde{\sigma} \mathbf{T}_{\alpha}(\Omega)-\Omega \mathbf{T}_{\alpha}(\widetilde{\delta})\right) /\left(\mho^{2}\right)\right)$

Furthermore, if $\Omega$ is differentiable, then $\mathbf{T}_{\alpha}(\Omega)$ $(\tau)=\tau^{1-\alpha}(d \Omega / d \tau)$.

Theorem 2 (see [12]). Suppose $\Omega:(0, \infty) \longrightarrow \mathbb{R}$ is a function such that $\Omega$ is differentiable and also $\alpha$-differentiable. Suppose $\sigma$ is a function defined in the range of $\Omega$ and also differentiable; then, one has the following rule:

$$
\mathbf{T}_{\alpha}(\Omega \mathrm{o} \sigma)(\tau)=\tau^{1-\alpha} \widetilde{\sigma}(\tau) \Omega^{\prime}(\widetilde{\sigma}(\tau)) .
$$

\section{Methods and Applications}

In this section, we present the first step of the TEM and the MTEM for finding analytical solutions of the CTFMNLSE defined as (2). For more details, see [5].

Suppose a CTFNLPDE

$$
\begin{array}{r}
\Gamma\left(\Phi, \Phi_{\tau}, \Phi_{\mathfrak{X}}, \mathbf{D}_{\tau}^{\alpha} \Phi, \mathbf{D}_{\mathfrak{X}}^{\beta} \Phi, \mathbf{D}_{\tau}^{2 \alpha}, \mathbf{D}_{\mathfrak{X}}^{2 \beta}, \ldots\right)=0, \\
0<\alpha \leq 1,0<\beta<1,
\end{array}
$$

where $\Phi$ and $\Gamma$ are an unknown function and a polynomial in its arguments, respectively.

Using a fractional travelling wave transformation

$$
\Phi(\mathfrak{X}, \tau)=\Lambda(\xi), \quad \xi=\mathfrak{X}-\frac{\mathscr{V}}{\alpha} \tau^{\alpha},
$$

where $\mathscr{V}$ is the velocity and substituting (7) into (6), we have a NLODE given by

$$
\Upsilon\left(\Lambda, \Lambda^{\prime}, \Lambda^{\prime \prime}, \Lambda^{\prime \prime \prime}, \ldots\right)=0,
$$

where' denotes the derivative with respect to $\xi$.

Here, since $\Psi=\Psi(\mathfrak{X}, \tau)$ in (2) is a complex function, for proceeding, we begin with the following travelling wave assumption:

$$
\Psi(\mathfrak{X}, \tau)=\Lambda(\xi) e^{i \psi},
$$

where $\xi=\eta\left(\mathfrak{X}-(\mathscr{V} / \alpha) \tau^{\alpha}\right)$ and $\psi=-\mathscr{K} \mathfrak{X}+(\mathfrak{X} / \alpha) \tau^{\alpha}+\zeta$, and $\zeta, \mathfrak{X}$, and $\mathscr{K}$ are parameters, representing the phase constant, frequency, and wave number, respectively. Substituting (9) into (2), we get real and imaginary parts as follows:

$$
\begin{gathered}
\eta^{2}\left(\sigma_{1}-3 \delta_{1} \mathscr{K}\right) \Lambda^{\prime \prime}+\left(\sigma_{2}+\left(\delta_{2}+\delta_{3}\right) \mathscr{K}\right) \Lambda^{3} \\
+\left(-P-\sigma_{1} \mathscr{K}^{2}+\delta_{1} \mathscr{K}^{3}-\delta_{4}\right) \Lambda=0 \\
\left(3 \delta_{1} \mathscr{K}^{2}-\mathscr{V}-2 \sigma_{1} \mathscr{K}\right) \Lambda^{\prime}-\delta_{1} \eta^{2} \Lambda^{\prime \prime \prime}+\left(\delta_{3}-\delta_{2}\right) \Lambda^{2} \Lambda^{\prime}=0 .
\end{gathered}
$$

Now, integrating the imaginary part of the equation and taking constant equal to zero, one may have

$$
3\left(3 \delta_{1} \mathscr{K}^{2}-\mathscr{V}-2 \sigma_{1} \mathscr{K}\right) \Lambda-3 \delta_{1} \eta^{2} \Lambda^{\prime \prime}+\left(\delta_{3}-\delta_{2}\right) \Lambda^{3}=0 .
$$

From (10) and (12), it can be followed that

$$
\begin{aligned}
\frac{\mathscr{K}^{3} \delta_{1}-\mathfrak{X}-\sigma_{1} \mathscr{K}^{2}-\delta_{4}}{3\left(3 \delta_{1} \mathscr{K}^{2}-\mathscr{V}-2 \sigma_{1}\right) \mathscr{K}} & =\frac{\sigma_{1}-3 \delta_{1} \mathscr{K}}{-3 \delta_{1}} \\
& =\frac{\mathscr{K} \delta_{2}+\mathscr{K} \delta_{3}+\sigma_{2}}{\delta_{3}-\delta_{2}} .
\end{aligned}
$$

From the above, it can be followed that

$$
\begin{aligned}
& \mathscr{V}=-\frac{\delta_{1} \mathfrak{X}+\delta_{1} \delta_{4}+2 \mathscr{K}\left(\sigma_{1}-2 \delta_{1} \mathscr{K}\right)^{2}}{\sigma_{1}-3 \delta_{1} \mathscr{K}}, \\
& \mathscr{K}=\frac{\sigma_{1}\left(\delta_{2}-\delta_{3}\right)-3 \sigma_{2} \delta_{1}}{6 \delta_{1} \delta_{2}} .
\end{aligned}
$$

Rewrite (10) into the following form:

$$
\begin{aligned}
\Lambda^{\prime \prime}+\lambda_{1} \Lambda^{3}-\lambda_{2} \Lambda & =0, \\
\operatorname{or} \Lambda^{\prime \prime} & =\lambda_{2} \Lambda-\lambda_{1} \Lambda^{3},
\end{aligned}
$$

where $\quad \lambda_{1}=\left(\sigma_{2}+\left(\delta_{2}+\delta_{3}\right) \mathscr{K}\right) /\left(\eta^{2}\left(\sigma_{1}-3 \delta_{1}\right)\right) \quad$ and $\lambda_{2}=-\left(-\mathfrak{X}-\sigma_{1} \mathscr{K}^{2}+\delta_{1} \mathscr{K}^{3}-\delta_{4}\right) /\left(\eta^{2}\left(\sigma_{1}-3 \delta_{1} \mathscr{K}\right)\right)$.

In the next two sections, we investigate the primary steps for detecting the exact solution of (10) by using the TEM and the MTEM.

The exact solution of (12) can be found in a similar way. 
3.1. Trial Equation Method(TEM). Firstly, CTFNLPDE (6) is reduced to NLODE (8) under transformation (7). Secondly, consider the trial equation

$$
\left(\Lambda^{\prime}\right)^{2}=\sum_{i=0}^{n} a_{i} \Lambda^{i}
$$

where $a_{i}$ and $n$ are constants, which are derived from the solution of the system and the balancing principle, respectively. Finally, the solution of (17) can be given by the integral form:

$$
\pm\left(\xi-\xi_{0}\right)=\int \frac{1}{\sqrt{\sum_{i=0}^{n} a_{i} \Lambda^{i}}} \mathrm{~d} \Lambda .
$$

Note 1. If

$$
\Lambda^{\prime \prime}=a_{0}+a_{1} \Lambda+\cdots+a_{n} \Lambda^{n}
$$

then integrating (19) with respect to $\xi$ once, we get

$$
\left(\Lambda^{\prime}\right)^{2}=\frac{2 a_{n}}{n+1} \Lambda^{n+1}+\cdots+a_{1} \Lambda^{2}+2 a_{0} \Lambda+d,
$$

Now, considering (16) and balancing $\Lambda^{\prime \prime}$ and $\Lambda^{3}$, we obtain $n=4$. So, the trial equation is

$$
\left(\Lambda^{\prime}\right)^{2}=a_{0}+a_{1} \Lambda+a_{2} \Lambda^{2}+a_{3} \Lambda^{3}+a_{4} \Lambda^{4},
$$

in which

$$
\left(\Lambda^{\prime}\right)^{2}=-\frac{\lambda_{1}}{2} \Lambda^{4}+\lambda_{2} \Lambda^{2}+d
$$

so we have

$$
a_{0}=d, a_{1}=0, a_{2}=\lambda_{2}, a_{3}=0, a_{4}=-\frac{\lambda_{1}}{2} .
$$

Now, (18) is rewritten with (23):

$$
\pm\left(\xi-\xi_{0}\right)=\int \frac{1}{\sqrt{a_{0}+\lambda_{2} \Lambda^{2}-\left(\lambda_{1} / 2\right) \Lambda^{4}}} \mathrm{~d} \Lambda .
$$

If we set $a_{0}=0$ in (24) and integrate this equation, the exact solution of (2) is obtained:

If $\lambda_{2}>0$, then we have bright and singular solutions, respectively:

where $n, a_{i}$, and integration constant $d$ are to be determined.

$$
\begin{aligned}
& \Psi(\mathfrak{X}, \tau)= \pm \sqrt{2\left(\lambda_{2} / \lambda_{1}\right)} e^{i\left(-\mathfrak{X} \mathscr{K}+(\mathfrak{X} / \alpha) \tau^{\alpha}+\zeta\right)} \operatorname{sech}\left(\sqrt{\lambda_{2}}\left(\eta\left(\mathfrak{X}-\frac{\mathscr{V}}{\alpha} \tau^{\alpha}\right)+\xi_{0}\right)\right), \\
& \Psi(\mathfrak{X}, \tau)= \pm \sqrt{-2 \frac{\lambda_{2}}{\lambda_{1}}} e^{i\left(-\mathfrak{X} \mathscr{K}+(\mathfrak{X} / \alpha) \tau^{\alpha}+\zeta\right)} \operatorname{csch}\left(\sqrt{\lambda_{2}}\left(\eta\left(\mathfrak{X}-\frac{\mathscr{V}}{\alpha} \tau^{\alpha}\right)+\xi_{0}\right)\right) .
\end{aligned}
$$

If $\lambda_{2}<0$, the singular periodic solutions appear as

$$
\begin{aligned}
& \Psi(\mathfrak{X}, \tau)= \pm \sqrt{2 \frac{\lambda_{2}}{\lambda_{1}}} e^{i\left(-\mathfrak{X} \mathscr{K}+(\mathfrak{X} / \alpha) \tau^{\alpha}+\zeta\right)} \sec \left(\sqrt{-\lambda_{2}}\left(\eta\left(\mathfrak{X}-\frac{\mathscr{V}}{\alpha} \tau^{\alpha}\right)+\xi_{0}\right)\right) \\
& \Psi(\mathfrak{X}, \tau)= \pm \sqrt{2 \frac{\lambda_{2}}{\lambda_{1}}} e^{i\left(-\mathscr{K} \mathfrak{X}+(\mathfrak{X} / \alpha) \tau^{\alpha}+\zeta\right)} \csc \left(\sqrt{-\lambda_{2}}\left(\eta\left(\mathfrak{X}-\frac{\mathscr{V}}{\alpha} \tau^{\alpha}\right)+\xi_{0}\right)\right) \\
& \pm\left(\xi-\xi_{0}\right)=\int \frac{F(\Lambda)}{G(\Lambda)} \mathrm{d} \Lambda .
\end{aligned}
$$

where $\xi_{0}$ is an arbitrary constant. For more details, see [13-15].

3.2. Modified Trial Equation Method (MTEM). In this section, instead of using (17), the trial equation can be chosen as

$$
\Lambda^{\prime}=\frac{F(\Lambda)}{G(\Lambda)}=\frac{a_{N} \Lambda^{N}+\cdots+a_{1} \Lambda+a_{0}}{b_{M} \Lambda^{M}+\cdots+b_{1} \Lambda+b_{0}}
$$

which is seen as the modified trial equation method when the same procedure is applied with the integral form solution of (29) as
If we now use trial (29) in (16) for balancing the procedure, we get $N-M=2$. Choosing $N=2$ and $M=0$, the trial equation is 


$$
\Lambda^{\prime}=\frac{a_{0}+a_{1} \Lambda+a_{2} \Lambda^{2}}{b_{0}}
$$

and we have

$$
\Lambda^{\prime \prime}=\frac{\left(a_{1}+2 a_{2} \Lambda\right)\left(a_{0}+a_{1} \Lambda+a_{2} \Lambda^{2}\right)}{b_{0}^{2}},
$$

where $a_{2} \neq 0$ and $b_{0} \neq 0$.

The corresponding system is

$$
\begin{aligned}
\frac{a_{0} a_{1}}{b_{0}^{2}} & =0, \\
\frac{2 a_{0} a_{2}+a_{1}^{2}}{b_{0}^{2}} & =\lambda_{2}, \\
3 a_{1} a_{2} b_{0}^{2} & =0, \\
\frac{2 a_{2}^{2}}{b_{0}^{2}} & =-\lambda_{1} .
\end{aligned}
$$

Solving the corresponding system, we obtain

$$
a_{0}= \pm i \frac{1}{2} b_{0}^{2} \sqrt{\frac{\lambda_{1}}{2}} \lambda_{2}, a_{1}=0, a_{2}= \pm i b_{0} \sqrt{\frac{\lambda_{1}}{2}}, b_{0}=b_{0} \text {. }
$$

Using these coefficients in (30), we get

$$
\pm\left(\xi-\xi_{0}\right)=\int \frac{b_{0}}{ \pm i(1 / 2) b_{0}^{2} \sqrt{\left(\lambda_{1} / 2\right)} \lambda_{2} \pm i b_{0} \sqrt{\left(\lambda_{1} / 2\right)} \Lambda^{2}} \mathrm{~d} \Lambda .
$$

When we integrate this equation and use the wave transformation, the exact travelling wave solutions of (2) are obtained as follows.

If $b_{0} \lambda_{1} \lambda_{2}<0$, then we have

$$
\begin{aligned}
& \Psi(\mathfrak{X}, \tau)= \pm \sqrt{\frac{-b_{0} \lambda_{2}}{2}} e^{i\left(-\mathscr{K} \mathfrak{X}+(\mathfrak{X} / \alpha) \tau^{\alpha}+\zeta\right)} \tanh \left(\frac{1}{2} \sqrt{-b_{0} \lambda_{1} \lambda_{2}}\left(\eta\left(\mathfrak{X}-\frac{\mathscr{V}}{\alpha} \tau^{\alpha}\right)+\xi_{0}\right)\right), \\
& \Psi(\mathfrak{X}, \tau)= \pm \sqrt{\frac{-b_{0} \lambda_{2}}{2}} e^{i\left(-\mathscr{K} \mathfrak{X}+(\mathfrak{X} / \alpha) \tau^{\alpha}+\zeta\right)} \operatorname{coth}\left(\frac{1}{2} \sqrt{-b_{0} \lambda_{1} \lambda_{2}}\left(\eta\left(\mathfrak{X}-\frac{\mathscr{V}}{\alpha} \tau^{\alpha}\right)+\xi_{0}\right)\right),
\end{aligned}
$$

and if $b_{0} \lambda_{1} \lambda_{2}>0$, then we have

$$
\begin{aligned}
& \Psi(\mathfrak{X}, \tau)= \pm \sqrt{\frac{b_{0} \lambda_{2}}{2}} e^{i\left(-\mathscr{K} \mathfrak{X}+(\mathfrak{X} / \alpha) \tau^{\alpha}+\zeta\right)} \tan \left(\frac{1}{2} \sqrt{b_{0} \lambda_{1} \lambda_{2}}\left(\eta\left(\mathfrak{X}-\frac{\mathscr{V}}{\alpha} \tau^{\alpha}\right)+\xi_{0}\right)\right), \\
& \Psi(\mathfrak{X}, \tau)= \pm \sqrt{\frac{b_{0} \lambda_{2}}{2}} e^{i\left(-\mathscr{K} \mathfrak{X}+(\mathfrak{X} / \alpha) \tau^{\alpha}+\zeta\right)} \cot \left(\frac{1}{2} \sqrt{b_{0} \lambda_{1} \lambda_{2}}\left(\eta\left(\mathfrak{X}-\frac{\mathscr{V}}{\alpha} \tau^{\alpha}\right)+\xi_{0}\right)\right),
\end{aligned}
$$

where $\xi_{0}$ is an arbitrary constant. For more details, see [16-18].

\section{Numerical Results in Tables and Charts}

Here, we let $\delta_{1}=\delta_{3}=\eta=1, \mathfrak{X}=\delta_{2}=\delta_{4}=\sigma_{2}=2, \zeta=\mathscr{V}=$ $0.5, \mathscr{K}=0.25, \sigma_{1}=-1, \alpha=0.90$, and $\xi_{0}=0$. Therefore, we have $\lambda_{1}=-1.57143$ and $\lambda_{2}=-2.24107$. Now, in the following sections, we calculate the exact solutions of CTFMNLSE (2) obtained through the two different methods presented in Section 3.

Figures 1(a), 1(c), 2(a), and 2(c) and also Figures 1(b), 1(d), 2(b), and 2(d) display 3D and 2D with the real part of solutions $\Psi_{1,1}, \Psi_{1,3}, \Psi_{2,1}$, and $\Psi_{2,3}$ obtained by the TEM and MTEM under the values presented in the above paragraph.
Tables 1-4 present the numerical results of analytical solutions of CTFMNLSE (2) obtained by the TEM and MTEM with several point sources through arbitrary. In addition, Figures 2-5 display 2D with the real part of solutions $\Psi_{1,1}$ and $\Psi_{2,1}$ for fixed $\mathfrak{X}=0.062$ and different values of $\alpha$. Figure 3 displays $3 \mathrm{D}$ with the imaginary and the real part of solutions $\Psi_{1,1}$ and $\Psi_{1,3}$. Also, Figure 6 displays $3 \mathrm{D}$ with the imaginary and real part of solution $\Psi_{2,1}$ and also $2 \mathrm{D}$ with the imaginary part of the solution $\Psi_{2,1}$ for fixed $\mathfrak{X}=0.062$ and different values of $\alpha$.

4.1. The Exact Solutions of CTFMNLSE (2) Obtained through the TEM. Considering the given values in Section 4 , we get Tables 1 and 2 . 


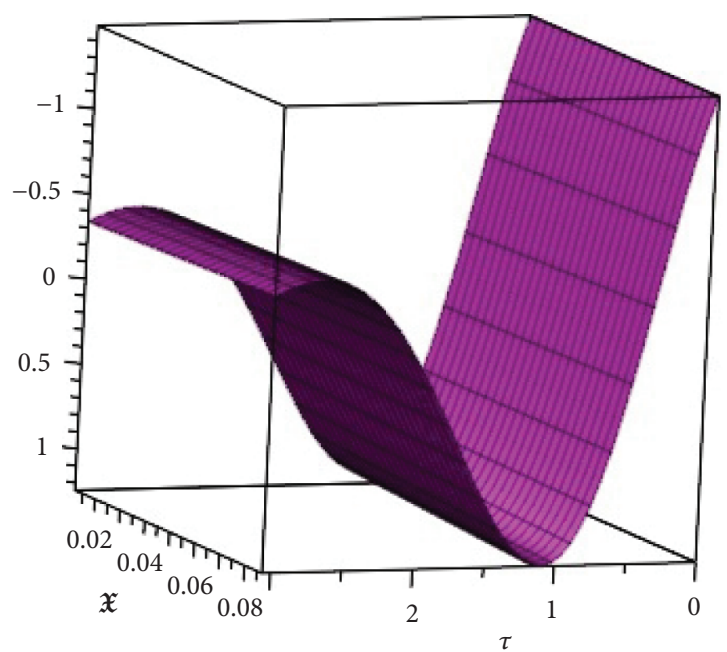

(a)

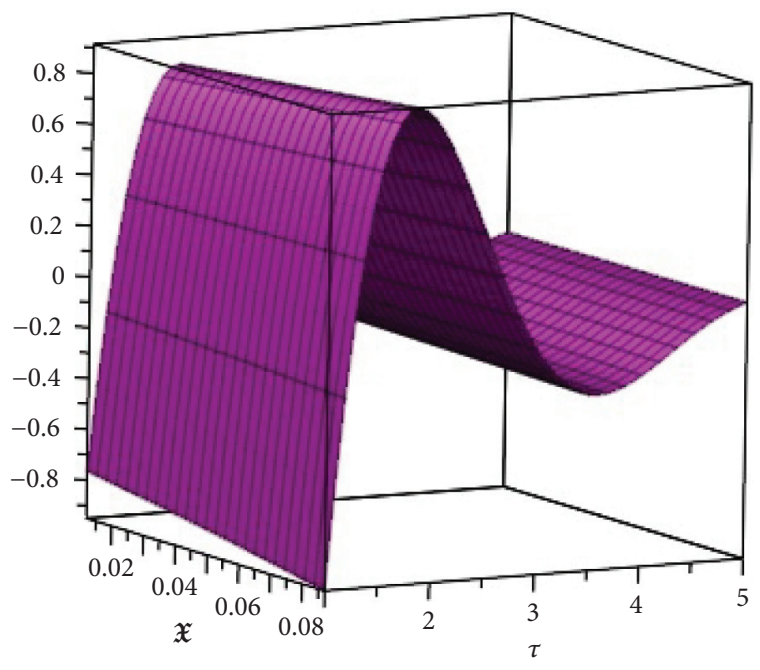

(c)

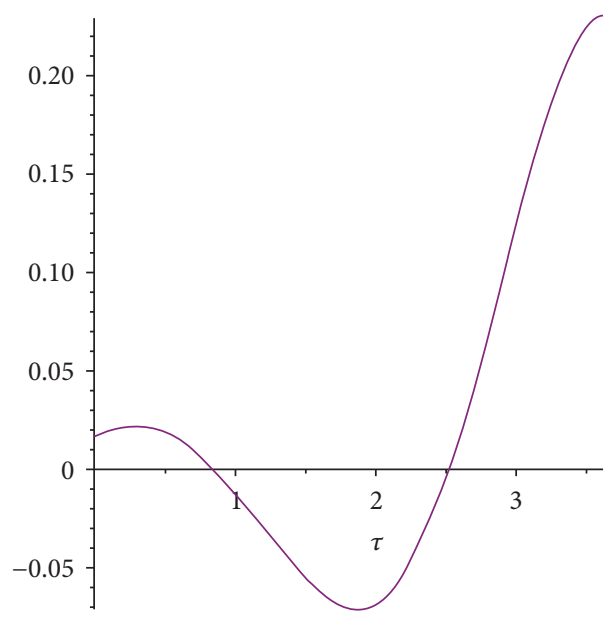

(b)

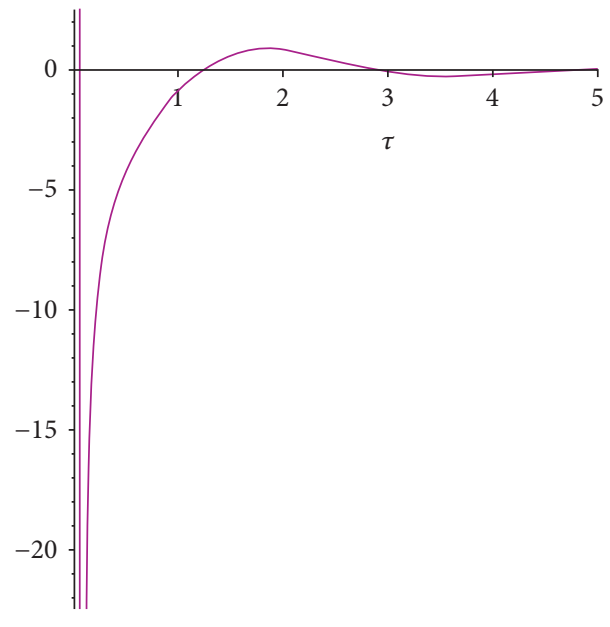

(d)

Figure 1: (a, b) 3D and 2D with the real part of solution $\Psi_{1,1}$. (c, d) 3D and 2D with the real part of solution $\Psi_{1,3}$ obtained via the TEM under the values presented in Section 4.

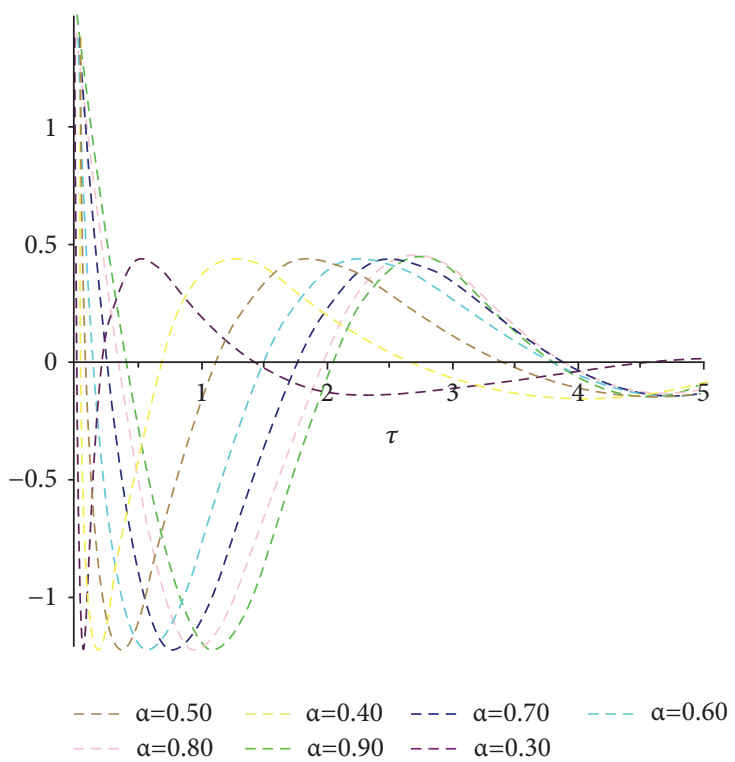

FIGURE 2: 2D with the real part of solution $\Psi_{1,1}$ for fixed $\mathfrak{X}=0.062$ and different values of $\alpha$. 
TABLE 1: The real part of exact solutions of CTFMNLSE (1) obtained by the TEM with several point sources through arbitrary.

\begin{tabular}{lccccc}
\hline $\mathfrak{X}$ & $\tau$ & $\Psi_{1,1}(\mathfrak{X}, \tau)$ & $\Psi_{1,2}(\mathfrak{X}, \tau)$ & $\Psi_{1,3}(\mathfrak{X}, \tau)$ & $\Psi_{1,4}(\mathfrak{X}, \tau)$ \\
\hline \multirow{2}{*}{0.012} & 0.012 & 1.44911 & -1.44911 & -351.38054 & -351.38054 \\
& 0.037 & 1.38186 & -1.38186 & -21.19424 & 39.15774 \\
0.037 & 0.062 & 1.31204 & -1.31204 & 21.46932 & 21.19424 \\
& 0.012 & 1.45334 & -1.45334 & 75.68599 & -21.46932 \\
& 0.037 & 1.38820 & -1.38820 & -83.68726 & -75.68599 \\
0.062 & 0.062 & 1.32018 & -1.32018 & 10.95095 & -10.95095 \\
& 0.012 & 1.45547 & -1.45547 & 18.97941 & -18.97941 \\
\hline
\end{tabular}

TABLE 2: The imaginary part of exact solutions of CTFMNLSE (1) obtained by the TEM with several point sources through arbitrary.

\begin{tabular}{lccccc}
\hline $\mathfrak{X}$ & $\tau$ & $\Psi_{1,1}(\mathfrak{X}, \tau)$ & $\Psi_{1,2}(\mathfrak{X}, \tau)$ & $\Psi_{1,3}(\mathfrak{X}, \tau)$ & $\Psi_{1,4}(\mathfrak{X}, \tau)$ \\
\hline \multirow{3}{*}{0.012} & 0.012 & 0.86568 & -0.86568 & -588.19565 & 558.19565 \\
& 0.037 & 0.96853 & -0.96853 & 26.26665 & -55.86884 \\
0.037 & 0.062 & 1.05867 & -1.05867 & -36.45427 & 36.26665 \\
& 0.012 & 0.85592 & -0.85592 & -109.43494 & 109.43494 \\
& 0.037 & 0.96009 & -0.96009 & 105.05287 & -105.05287 \\
0.062 & 0.062 & 1.05167 & -0.84497 & -18.86303 & 18.86303 \\
& 0.012 & 0.84497 & -0.95027 & -27.81244 & 27.81244 \\
& 0.037 & 0.95027 & -1.04316 & -53.37174 & 53.37174 \\
\hline
\end{tabular}

TABLE 3: The real part of exact solutions of CTFMNLSE (1) obtained by the MTEM with several point sources through arbitrary.

\begin{tabular}{lccccc}
\hline $\mathfrak{X}$ & $\tau$ & $\Psi_{2,1}(\mathfrak{X}, \tau)$ & $\Psi_{2,2}(\mathfrak{X}, \tau)$ & $\Psi_{2,3}(\mathfrak{X}, \tau)$ & -351.20377 \\
\hline \multirow{3}{*}{0.012} & 0.012 & -0.00084 & 0.00084 & 39.17921 & 351.20377 \\
& 0.037 & 0.00941 & -0.00941 & 21.20630 & -39.17921 \\
& 0.062 & 0.02081 & -0.02081 & -21.47600 & -21.20630 \\
0.037 & 0.012 & -0.01342 & 0.01342 & -75.67674 & 75.67674 \\
& 0.037 & -0.00479 & 0.00479 & 83.77139 & -83.77139 \\
0.062 & 0.062 & 0.00519 & -0.00519 & -10.95634 & 10.95634 \\
& 0.012 & -0.02575 & 0.02575 & -18.98482 & 18.98482 \\
& 0.037 & -0.01873 & 0.01873 & -41.97342 & 41.97342 \\
\hline
\end{tabular}

TABLE 4: The imaginary part of exact solutions of CTFMNLSE (1) obtained by the MTEM with several point sources through arbitrary.

\begin{tabular}{lccccc}
\hline $\mathfrak{X}$ & $\tau$ & $\Psi_{2,1}(\mathfrak{X}, \tau)$ & $\Psi_{2,2}(\mathfrak{X}, \tau)$ & $\Psi_{2,3}(\mathfrak{X}, \tau)$ & $\Psi_{2,4}(\mathfrak{X}, \tau)$ \\
\hline \multirow{3}{*}{0.012} & 0.012 & 0.00140 & -0.00140 & 587.89974 & -587.89974 \\
& 0.037 & -0.01342 & 0.01342 & -55.89947 & 55.89947 \\
0.037 & 0.062 & -0.02579 & 0.02579 & -26.28158 & 26.28158 \\
& 0.012 & 0.02279 & -0.02279 & 36.46563 & -36.46563 \\
& 0.037 & 0.00691 & -0.00691 & 109.42158 & -109.42158 \\
0.062 & 0.062 & -0.00651 & 0.00651 & -105.15849 & -18.87231 \\
& 0.012 & 0.04436 & -0.04436 & 27.82036 & -27.82036 \\
& 0.037 & 0.02745 & -0.02745 & 53.37043 & -53.37043 \\
\hline
\end{tabular}




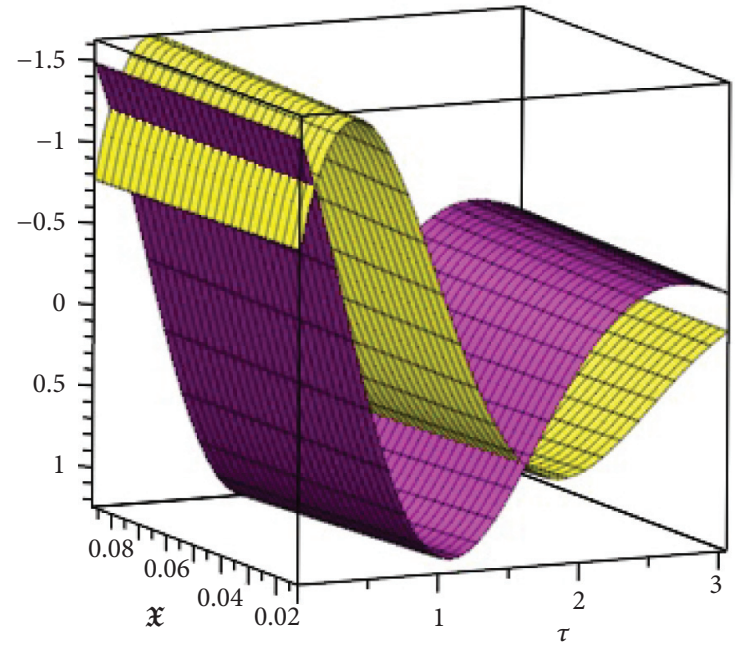

(a)

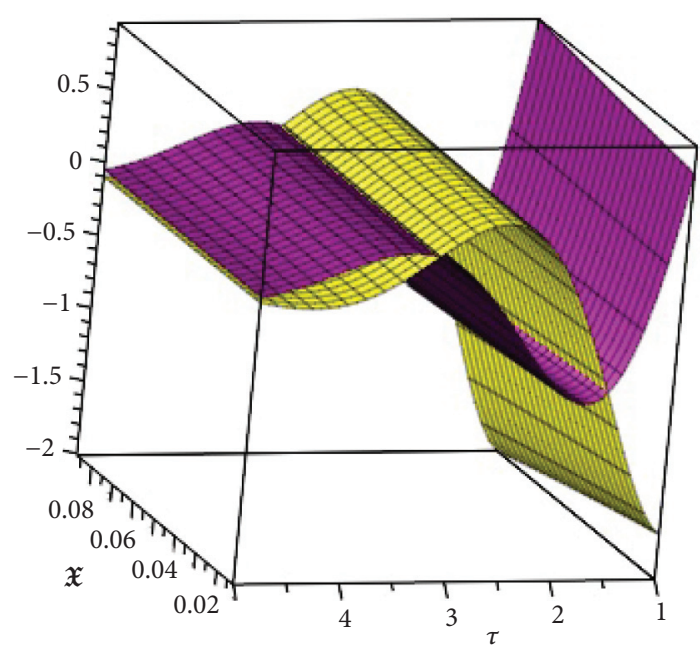

(b)

FIGURE 3: 3D with the imaginary and the real part of solutions $\Psi_{1,1}$ and $\Psi_{1,3}$.

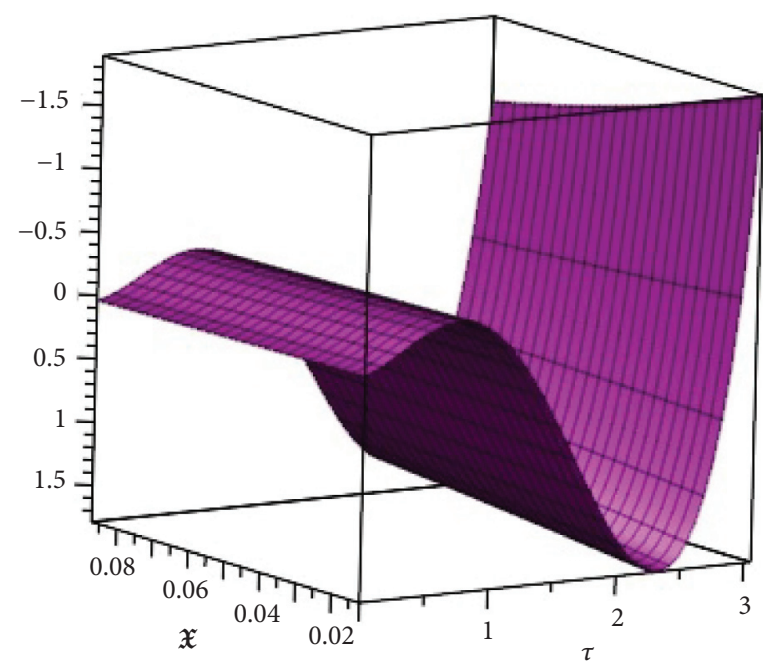

(a)

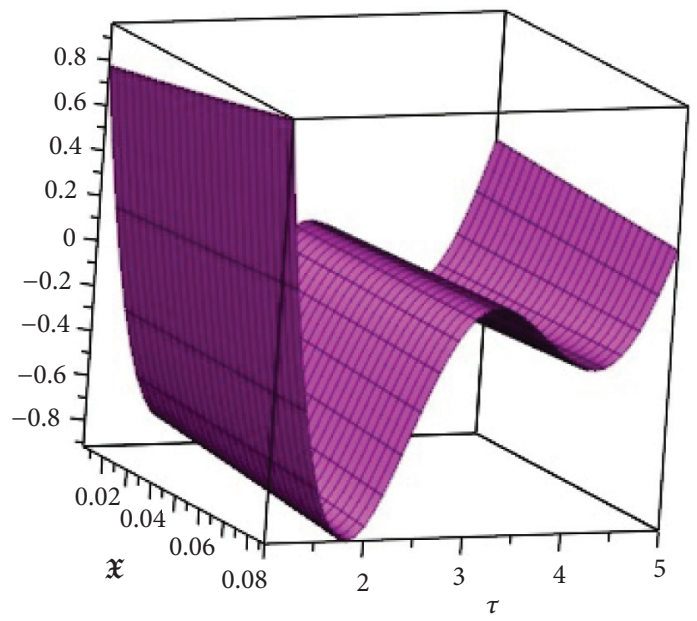

(c)

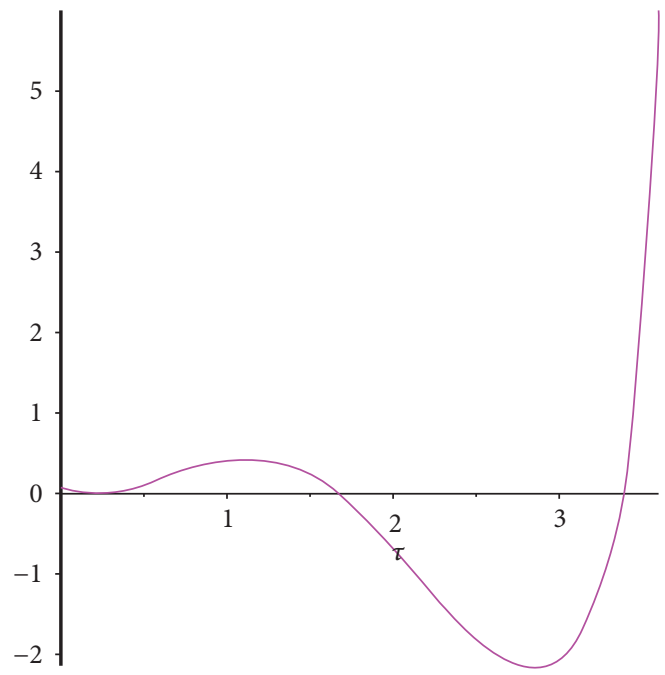

(b)

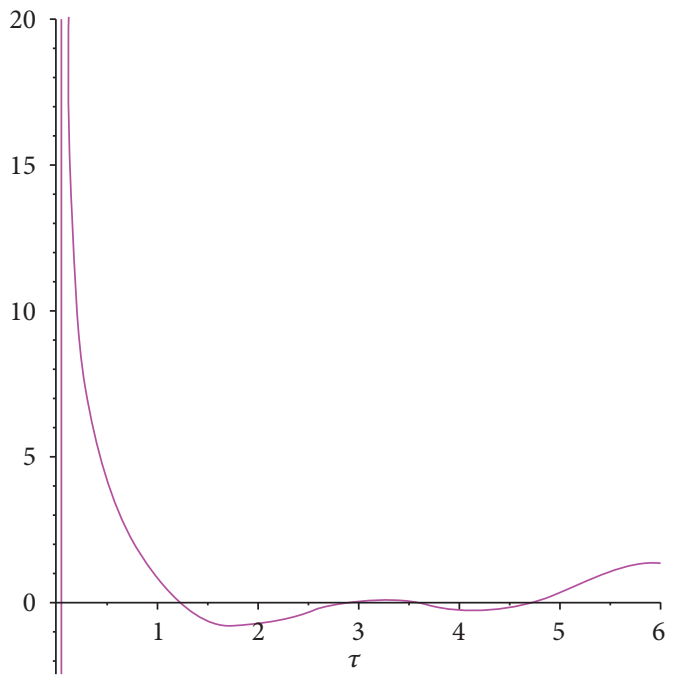

(d)

Figure 4: (a, b) 3D and 2D with the real part of solution $\Psi_{2,1}$ (c, d) 3D and 2D with the real part of solution $\Psi_{2,3}$ obtained via the MTEM under the values presented in Section 4. 


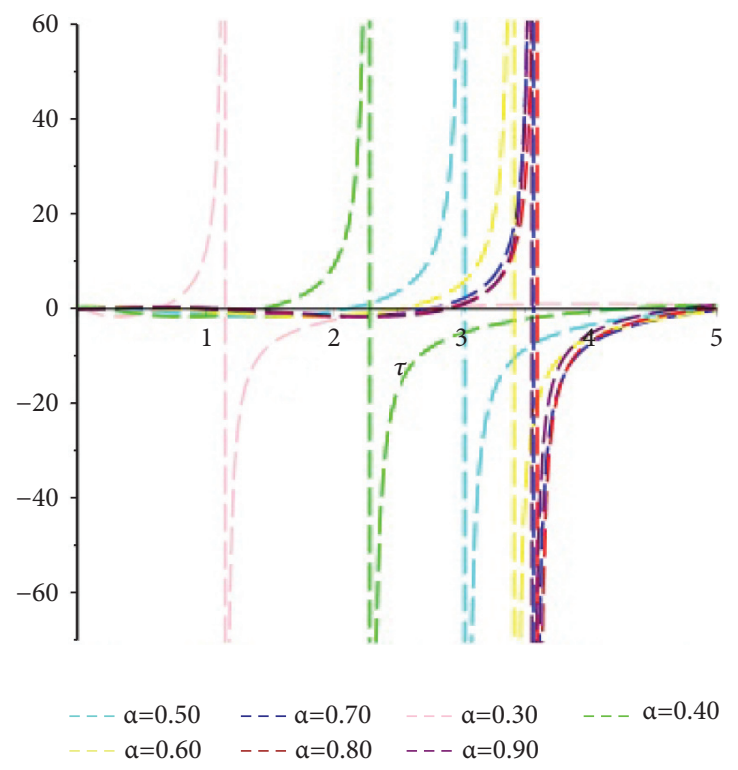

Figure 5: 2D with the real part of the solution $\Psi_{2,1}$ for fixed $\mathfrak{X}=0.062$ and different values of $\alpha$.
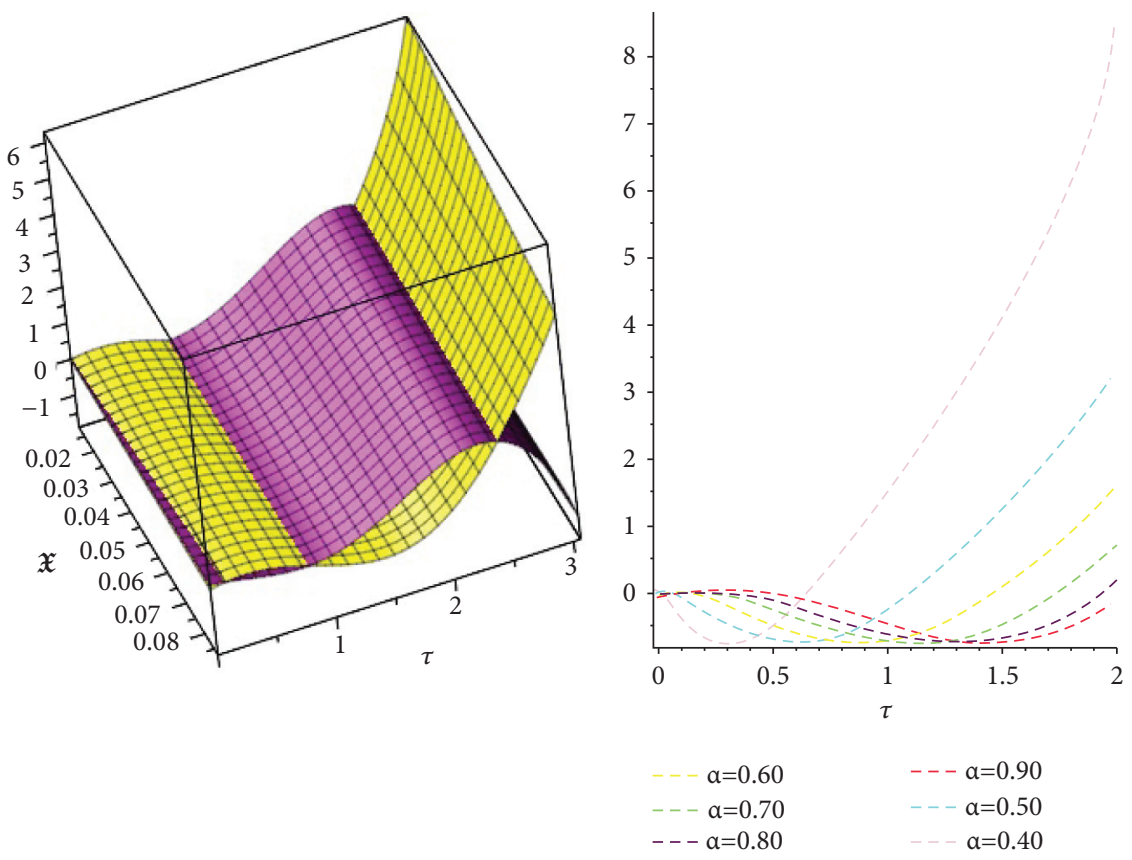

(a)

(b)

Figure 6: 3D with the imaginary and the real part of solutions $\Psi_{2,1}$ and also 2D with the imaginary part of the solution $\Psi_{2,1}$ for fixed $\mathfrak{X}=0.062$ and different values of $\alpha$.

TABLE 5: Calculation of the difference between solutions $\Psi_{1,1}$ and also $\Psi_{2,1}$, represented by $\Delta_{\text {TEM }}$ and $\Delta_{\text {MTEM }}$, for different values of $\mathfrak{X}$ and $\tau$.

\begin{tabular}{|c|c|c|c|c|c|c|c|c|c|}
\hline & \multicolumn{9}{|c|}{$\tau=0.012,0.037$, and 0.062} \\
\hline & \multicolumn{3}{|c|}{$\mathfrak{X}=0.012$} & \multicolumn{3}{|c|}{$\mathfrak{X}=0.037$} & \multicolumn{3}{|c|}{$\mathfrak{X}=0.062$} \\
\hline$\Delta_{\mathrm{TEM}}$ & 0.33695 & 0.13707 & 0.06982 & 0.06514 & 0.13316 & 0.06802 & 0.06293 & 0.12905 & 0.06612 \\
\hline$\Delta_{\mathrm{MTFM}}$ & 0.01025 & 0.02165 & 0.01140 & 0.00863 & 0.01861 & 0.00998 & 0.00792 & 0.01556 & 0.00854 \\
\hline
\end{tabular}




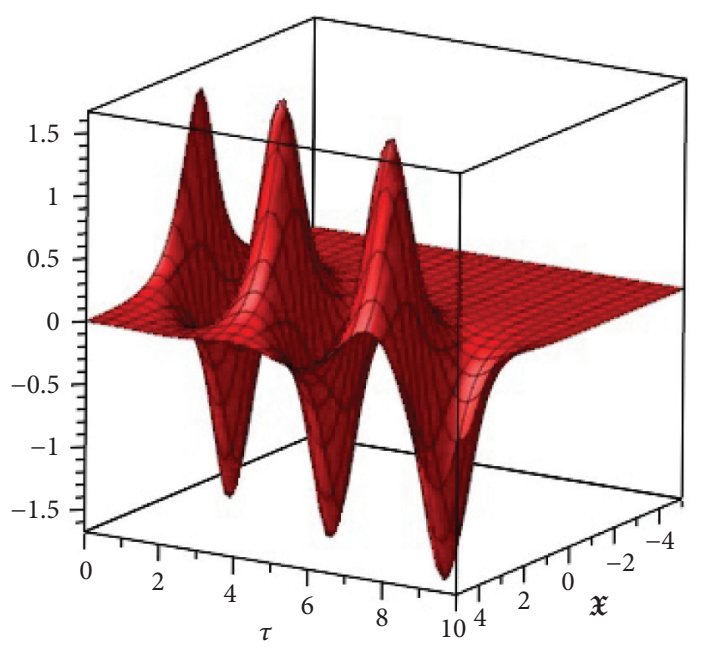

(a)

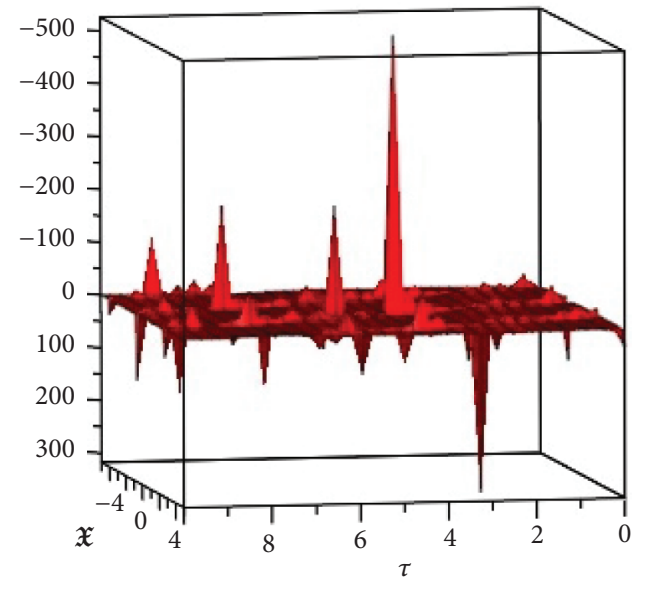

(c)

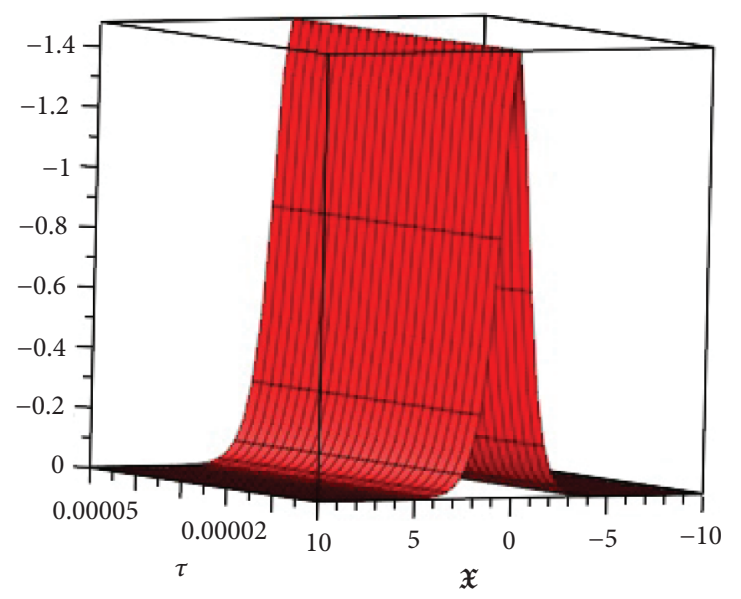

(e)

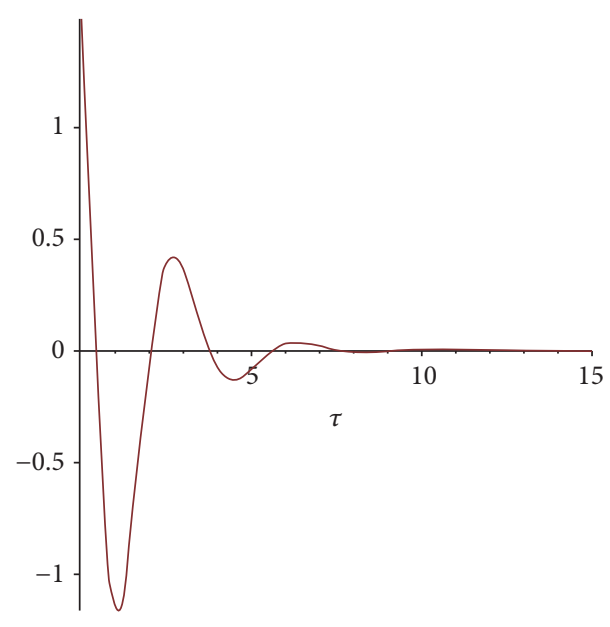

(b)

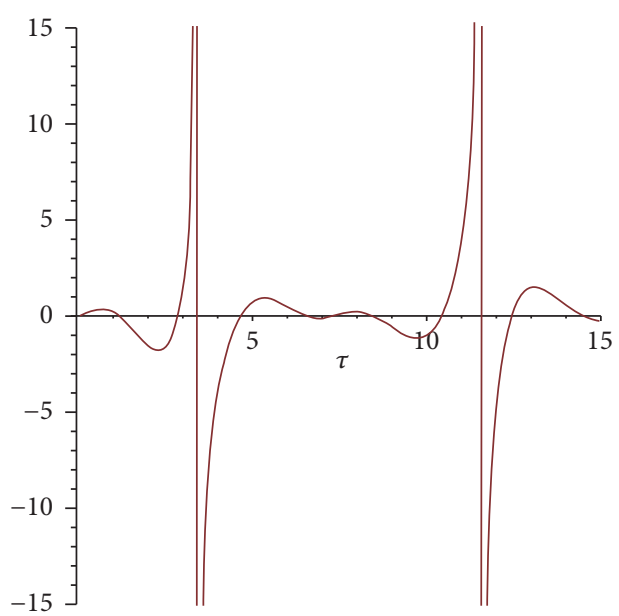

(d)

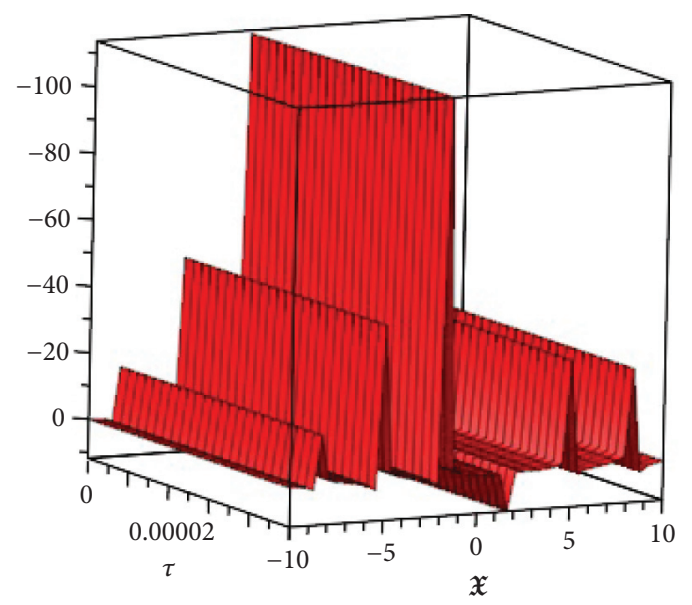

(f)

FIgure 7: (a, e, b) 3D and 2D with the real part of solutions $\Psi_{1,1}$ obtained via the TEM under the values presented in Section 4. (c, f, d) 3D and $2 \mathrm{D}$ with the real part of solutions $\Psi_{2,1}$ obtained via the MTEM under the values presented in Section 4. 


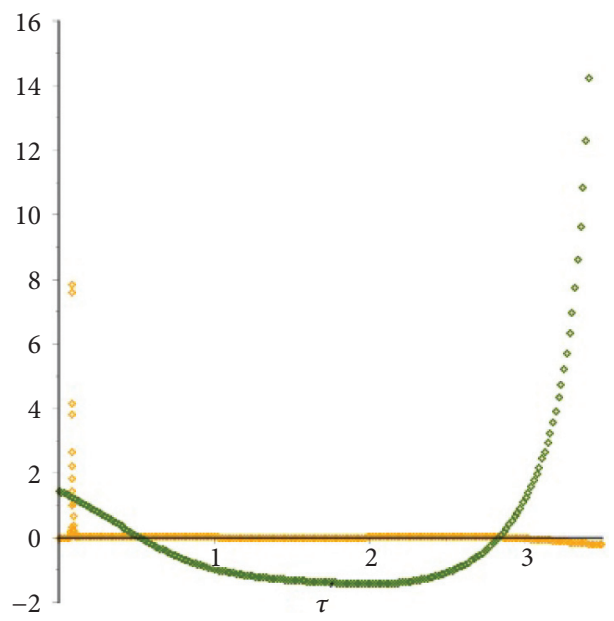

FIGURE 8: 2D with the differences between the real part of solutions obtained through the TEM and MTEM for fixed $\mathfrak{X}=0.062$ and $\alpha=0.90$.

4.2. The Exact Solutions of CTFMNLSE (2) Obtained through the MTEM. Considering the given values in Section 4, we get Tables 3 and 4 .

\section{Concluding Remarks}

Using the TEM and MTEM, firstly, we found the exact solutions of CTFMNLSE (2), and finally, we presented numerical results in tables and charts.

According to Table 5, we can observe that the differences between solutions $\Psi_{1,1}$ and also $\Psi_{2,1}$ for fixed $\mathfrak{X}$ and different values of $\tau$ are considerable. It is clear that these differences obtained through the MTEM are less than those of the TEM. In other words, for fixed $\mathfrak{X}$, by changing the value of $\tau$, the MTEM results in more minor changes than the TEM. Also, based on Figure 7, we can observe that the results gained by the MTEM have higher accuracy than the TEM.

Figure 8 displays the differences between the real part of solutions obtained by the TEM and MTEM for fixed $\mathfrak{X}=0.062$ and $\alpha=0.90$. As you can see, the difference obtained among $\Psi_{1,3}, \Psi_{1,4}$ and $\Psi_{2,3}, \Psi_{2,4}$ is more minor than the difference obtained among $\Psi_{1,1}, \Psi_{1,2}$ and $\Psi_{2,1}, \Psi_{2,2}$.

\section{Data Availability}

No data were used to support this study.

\section{Conflicts of Interest}

The authors declare that there are no conflicts of interest.

\section{References}

[1] T. Cazenave, An Introduction to Nonlinear Schrödinger Equations, Universidade Federal do Rio de Janeiro, Centro de Ciências Matemáticas e da, Rio de Janeiro, Brazil, 1989.

[2] M. Eslami, H. Rezazadeh, M. Rezazadeh, and S. S. Mosavi, "Exact solutions to the space-time fractional Schrödinger-Hirota equation and the space-time modified KDV-Zakharov-Kuznetsov equation," Optical and Quantum Electronics, vol. 49, no. 8, 2017.
[3] M. T. Darvishi, M. Najafi, and A.-M. Wazwaz, "Some optical soliton solutions of space-time conformable fractional Schrödinger-type models and their traveling wave solutions," Physica Scripta, vol. 96, no. 6, 2021.

[4] H. Rezazadeh, Mirhosseini-Alizamini, S. Mehdi et al., "New optical solitons of nonlinear conformable fractional Schrödinger-Hirota equation," Optik, vol. 172, 2018.

[5] U. Younas, M. Younis, A. R. Seadawy, S. T. R. Rizvi, S. Althobaiti, and S. Sayed, "Diverse exact solutions for modified nonlinear Schrodinger equation with conformable fractional derivative," Results in Physics, vol. 20, Article ID 103766, 2021.

[6] B.-H. Wang, Y.-Y. Wang, C.-Q. Dai, and Yi-X. Chen, "Dynamical characteristic of analytical solitons for the space-time fractional Fokas-Lenells equation," Alexandria Engineering Journal, vol. 59, no. 6, 2020.

[7] G.-Z. Wu, Li-J. Yu, and Y.-Y. Wang, "Fractional optical solitons of the space-time fractional nonlinear Shrodinger equation," Optik - International Journal for Light and Electron Optics, Elsevier, vol. 207, , Article ID 164405, 2020.

[8] Li-J. Yu, G.-Z. Wu, Y.-Y. Wang, and Yi-X. Chen, "Traveling wave solutions constructed by Mittag-Leffler function of a (2+1)-dimensional space-time fractional NLS equation," Results in Physics, vol. 17, Article ID 103156, 2020.

[9] J. E. Macías-Díaz, M. G. Medina-Guevar, and H. . VargasRodríguez, "Exact solutions of non-linear Klein-Gordon equation with non-constant coefficients through the trial equation method," Journal of Mathematical Chemistry, vol. 59, 2021.

[10] T. D. Şeyma and S. Duman, "The modified trial equation method to the Van der Waals Model," Afyon Kocatepe University Journal of Sciences and Engineering, vol. 21, pp. 266-272, Article ID 021301, 2021.

[11] Demiray, T. şeyma, and S. Duman, "The modified trial equation method to the $(2+1)$-dimensional Broer-KaupKupershmidt equation and Kolmogorov-Petrovskii-Piskunov equation," Balıkesir Üniversitesi Fen Bilimleri Enstitüsü Dergisi, vol. 2, no. 23, pp. 673-684, 2021.

[12] R. Khalil, M. Al Horani, A. Yousef, and M. Sababheh, "A new definition of fractional derivative," Journal of Computational and Applied Mathematics, 2014.

[13] Li Yang, "Trial equation method for exact travelling solutions of fifth order Caudrey- Dodd-Gibbon equation," Scholars 
Journal of Engineering and Technology (SJET), vol. 2, no. 3C, pp. 472-476, 2014.

[14] J.-Y. Hu, X.-B. Feng, and Y.-F. Yang, "Optical envelope patterns perturbation with full nonlinearity for Gerdjikov-Ivanov equation by trial equation method," Optik, vol. 157, 2021.

[15] Y. Yıldırım, "Optical soliton molecules of Lakshmanan-Porsezian-Daniel model in birefringent fibers by trial equation technique," Optik, vol. 203, Article ID 162690, 2020.

[16] H. Bulut and Y. Pandir, "Modified trial equation method to the nonlinear fractional Sharma-Tasso-Olever equation," International Journal of Modeling and Optimization, vol. 3, 2013.

[17] M. Odabasi and E. Misirli, "On the solutions of the nonlinear fractional differential equations via the modified trial equation method," Mathematical Methods in the Applied Sciences, vol. 41, no. 3, pp. 904-911, 2018.

[18] H. Bulut, G. . Yel, and H. M. Baskonus, "Novel structure to the coupled nonlinear Maccari"s system by using modified trial equation method," Advanced Mathematical Models \& Applications, vol. 2, no. 1, 2017. 